\title{
ON FOURIER IMPLEMENTATION OF TOLERANCE MODELING OF LINEAR ELASTODYNAMICS OF PERIODIC COMPOSITES
}

\author{
Dorota Kula \\ Faculty of Civil and Environmental Engineering \\ Warsaw University of Life Sciences - SGGW, Poland \\ kuladorota@wp.pl
}

\begin{abstract}
The report presents a new model of linear elastodynamics of periodic composites. The study deals with a certain extension of the ideas yield tolerance modelling. In the proposed model the displacement field is determined by an infinite number of unknowns. This disappointment seems to be apparent in many cases of periodicity since the sequence of tolerance shape functions used in the modelling procedure is not unique and opens the possibility to control the model equations. It has been formulated and solved, within the framework of the resulting model, the problem of independence short-term and long-term perturbations by linearly elastic composite solid.
\end{abstract}

Keywords: tolerance averaging, linear elastodynamics, periodic composites, boundary effect behaviour, short-wave perturbations

\section{Formulation of the problem}

The subject of consideration is a periodic linearly elastic composite. Material properties of the composite are described by the elastic modulus tensor represented in the distinguished Carthesian coordinate system by $\mathbf{A}=\left(C_{i j k l}\right), i, j, k, l=1,2,3$, and by mass density $\rho$, which are $\Delta$-periodic fields with respect to the related periodicity cell $\Delta \subset R^{m}$. In the sequel $\lambda \equiv \operatorname{diam}(\Delta)$ will be referred to as the microstructural parameter. The number of periodicity direction is denoted here by $m$ and hence $m$ is an arbitrary positive integer less than 3. Denoting body forces by $\mathbf{b}$ we can rewrite first type linear elasticity initial-boundary value problem by

$$
\begin{aligned}
& \rho \ddot{\mathbf{w}}-\nabla^{T}(\mathbf{A}[\mathbf{E}])=\mathbf{b} \\
& \mathbf{w}=\left.\mathbf{w}(z, t)\right|_{z \in \partial \Omega}=\mathbf{w}_{\partial}(z, t) \\
& \left.\mathbf{u}(z, t)\right|_{t=0}=\mathbf{u}_{0}(z)
\end{aligned}
$$

where

$$
\mathbf{E}=\frac{1}{2}\left(\nabla \mathbf{w}+\nabla^{T} \mathbf{w}\right)
$$


To equations (1) the continuity condition for stresses of normal interfaces $\Gamma$ between components should be attached. It will be formalized in the form

$$
[\mathbf{q}](\nabla \mathbf{w})=\left(\mathbf{n}^{T} \mathbf{A} \nabla \mathbf{w}\right)_{+}-\left(\mathbf{n}^{T} \mathbf{A} \nabla \mathbf{w}\right)_{-}=\mathbf{0}
$$

which should be satisfied almost everywhere in $\Gamma$. In (2) indices "+" and "_"“ identify different sides of interfaces between components.

Material properties of the linear elastic composite under consideration is described by the stiffness modulus $\mathbf{A} \equiv\left(C_{i j k l}\right), i, j, k, l=1,2,3$, and the mass density $\rho=\rho(z), z \in R^{3}$. Fields $C_{i j k l}=C_{i j k l}(x)$ and $\rho=\rho(x)$ should be $\Delta$-periodic with respect to the distinguished periodicity cell $\Delta \subset R^{m}$. Symbol $x \in R^{m}, m=1,2$, denotes here the orthogonal projection of $z \in R^{3}$ onto the periodicity directions space being a certain linear subspace of $R^{3}$. At the same time $y \in R^{3-m}$ describes directions of $z \in R^{3}$ perpendicular to the periodicity directions. Hence $z \equiv(x, y) \in R^{3}$. Symbol $\mathbf{b} \in R^{3}$ denotes body forces.

Every decomposition $\mathbf{w}=\mathbf{w}_{\text {long }}+\mathbf{w}_{\text {short }}$, in which $\mathbf{w}_{\text {short }}$ denotes a certain countable sum of various-periodic partial displacements such that $\left(\mathbf{n}^{T} \mathbf{A} \nabla \mathbf{w}_{\text {short }}\right)_{+}=\left(\mathbf{n}^{T} \mathbf{A} \nabla \mathbf{w}_{\text {short }}\right)_{-}=\mathbf{0}$ and $\mathbf{w}_{\text {long }} \equiv \mathbf{w}-\mathbf{w}_{\text {short }}$ will be referred to as a short-long wave decomposition of the displacement field. In every such decomposition $\langle\mathbf{w}\rangle=\mathbf{w}_{\text {long }}$ and hence $\mathbf{w}_{\text {long }}$ represents an averaged displacement field.

If in the framework of the model of linear elasticity (exact or averaged) $1^{\circ}$ via basic unknowns of which terms $\mathbf{w}_{\text {short }}$ and $\mathbf{w}_{\text {long }}$ can be determined the model will be referred to as a shortwave - longwave model. Moreover, if in the framework of this model $2^{\circ}$ equations of this model can be separated into two different sets of equations the first or second of which exclusively describes one part $\mathbf{w}_{\text {short }}$ or $\mathbf{w}_{\text {long }}$ of the displacement field then we will say that the composite moves (in the framework of this model) impaired long-and short-wave perturbations regardless. This property is independent on the body force field $\mathbf{b}$.

It must be emphasized that the existence of the shortwave - longwave model is an individual property of the composite.

The aim of the paper is to obtain the shortwave - longwave model of the linear elasticity. This model will be in fact a certain extension of the averaged model of elastodynamics, built and repeatedly discussed by Woźniak, cf. [1-3].

\section{Tolerance averaging}

In the framework of the tolerance averaging approach the displacement field is investigated in the form of the well-known micro-macro decomposition

$$
\mathbf{w} \approx \mathbf{w}_{M} \equiv \mathbf{w}_{0}(z)+h^{A}(x) \psi_{\mathrm{A}}(z)
$$


in which fields $\mathbf{w}_{0}=\mathbf{w}_{0}(z)$ and $\psi_{\mathrm{A}}=\psi_{\mathrm{A}}(z), A=1, \ldots, N, z \in R^{3}$ referred to as the averaged displacement field and the fluctuation amplitude field, respectively, are basic unknowns of the resulting tolerance model. Functions $h^{A}=h^{A}(x)$ are shape functions here which are $\Delta$-periodic and should be specified in every special problem. Basic unknowns $\mathbf{w}_{0}=\mathbf{w}_{0}(z)$ and $\psi_{\mathrm{A}}=\psi_{\mathrm{A}}(z)$ as well as the finite sequence of shape functions $h^{A}=h^{A}(x)$ should satisfy certain additional conditions. In the framework of this paper the most important are $\left\langle h^{A} \rho\right\rangle=0$ and $\left\langle h^{A} \mathbf{A}\right\rangle=0$. For particulars the reader is referred to [1-3].

In the framework of the refined tolerance model which we are to introduce in this paper we shall assume that the residual displacement field defined as $\mathbf{w}_{\text {res }} \equiv \mathbf{w}-\mathbf{w}_{M}$ can be represented as the Fourier expansion

$$
\mathbf{w}_{r e s}(x, y, t)=\sum_{k=0}^{+\infty} \mathbf{a}_{k}(x, y, t) \varphi^{k}(x)
$$

with respect to the Fourier basis $\varphi^{r}=\varphi^{r}(x)$. To this and we shall assume that the finite sequence of shape functions $h^{A}=h^{A}(x)$ is supported by the additional condition

$$
[\mathbf{q}]\left(\nabla \mathbf{w}_{r e s}\right)=\left(\mathbf{n}^{T} \mathbf{A} \nabla \mathbf{w}_{r e s}\right)_{+}=\left(\mathbf{n}^{T} \mathbf{A} \nabla \mathbf{w}_{r e s}\right)_{-}=\mathbf{0}
$$

Hence in the framework of the refined tolerance model the displacement field will be investigated in the form of the decomposition

$$
\mathbf{w}=\mathbf{w}_{\text {long }}+\mathbf{w}_{\text {short }}
$$

in which

$$
\begin{aligned}
& \mathbf{w}_{\text {long }}=\mathbf{u}+\lambda g^{A} \Psi_{\mathrm{A}} \\
& \mathbf{w}_{\text {short }}=\mathbf{a}_{p} \varphi^{p}(x) \equiv \mathbf{a}_{1}(x, y, t) \varphi^{1}(x)+\mathbf{a}_{2}(x, y, t) \varphi^{2}(x)+\ldots
\end{aligned}
$$

where $\mathbf{u} \equiv \mathbf{w}_{0}+\mathbf{a}_{0} \varphi^{0}$ and $h^{A}(x) \equiv \lambda g^{A}\left(\frac{x}{\lambda}\right)$ for $x \in R^{m}$. Here $p=1,2, \ldots$ and in the sequel $p, q, r=1,2, \ldots$. Under the assumption (6) the decomposition (7) is a proper decomposition of the displacement field onto shortwave and longwave parts defined in the first section.

To make the choice of shape functions and Fourier basis independent on the material properties of the composite components we shall restrict the Fourier basis $\varphi^{p}$ to that which satisfy condition

$$
\left.\nabla \varphi^{p}\right|_{\Gamma}=0
$$


stronger than (6). It yields

$$
\left\langle c \varphi^{p}\right\rangle=0 \quad\left\langle K \varphi^{p}\right\rangle=0 \quad\left\langle g^{A} c\right\rangle=0 \quad\left\langle g^{A} K\right\rangle=0
$$

The formal realization of the tolerance modeling

$$
\begin{aligned}
& \langle\rho \ddot{\mathbf{w}}\rangle-\nabla^{T}(\langle\mathbf{A}\rangle[\nabla \mathbf{w}])=\langle b\rangle \\
& \left\langle g^{A} \rho \ddot{\mathbf{w}}\right\rangle-\left\langle g^{A} \nabla^{T}(\mathbf{A}[\nabla \mathbf{w}])=\left\langle g^{A} b\right\rangle\right. \\
& \left\langle\varphi^{p} \rho \ddot{\mathbf{w}}\right\rangle-\left\langle\varphi^{p} \nabla^{T}(\mathbf{A}[\nabla \mathbf{w}])=\left\langle\varphi^{p} b\right\rangle\right.
\end{aligned}
$$

leads, under denotations $\nabla_{p e r} \equiv\left[\partial_{1}, \ldots, \partial_{m}, 0, \ldots, 0\right]$ and $\nabla_{p e r} \equiv\left[0, \ldots, 0, \partial_{m+1}, \ldots, \partial_{3}\right]$, to the refined tolerance model equations

$$
\begin{aligned}
& \langle\rho\rangle \ddot{\mathbf{u}}-\left\langle\nabla^{T}\{\langle\mathbf{A}\rangle[\nabla \mathbf{w}]\}-\left\langle\nabla^{T}\left\{\left\langle\mathbf{A}\left[\nabla g^{A}\right]\right\rangle \psi_{A}\right\}\right\rangle-\left\langle\nabla^{T}\left\{\left\langle\mathbf{A}\left[\nabla \varphi^{p}\right]\right\rangle \mathbf{a}_{p}\right\}\right\rangle=\langle\mathbf{b}\rangle\right. \\
& \left(\left[\begin{array}{cc}
\lambda^{2}\left\langle\rho g^{A} g^{B}\right\rangle & \lambda\left\langle\rho g^{A} \varphi^{q}\right\rangle \\
\lambda\left\langle\rho \varphi^{p} g^{B}\right\rangle & \left\langle\rho \varphi^{p} \varphi^{q}\right\rangle
\end{array}\right]\left[\begin{array}{c}
\ddot{\Psi}_{B} \\
\ddot{\mathbf{a}}_{q}
\end{array}\right]-\nabla^{T} \Phi\left(\left[\begin{array}{cc}
\lambda^{2}\left\langle g^{A} g^{B} \mathbf{A}\right\rangle & \lambda\left\langle g^{A} \varphi^{q} \mathbf{A}\right\rangle \\
\lambda\left\langle\varphi^{p} g^{A} \mathbf{A}\right\rangle & \left\langle\varphi^{p} \varphi^{q} \mathbf{A}\right\rangle
\end{array}\right] \nabla^{T} \Phi\left[\begin{array}{c}
\psi \\
\mathbf{a}_{q}
\end{array}\right]\right)+\right. \\
& +\left(\left[\begin{array}{cc}
\lambda\left\langle g^{A} \nabla_{p e r}^{T} g^{B} \mathbf{A}\right\rangle & \lambda\left\langle g^{A} \nabla_{p e r}^{T} \varphi^{q} \mathbf{A}\right\rangle \\
\lambda\left\langle\varphi^{p} \nabla_{p e r}^{T} g^{B} \mathbf{A}\right\rangle & \left\langle\varphi^{p} \nabla_{p e r}^{T} \varphi^{q} \mathbf{A}\right\rangle
\end{array}\right]-\left[\begin{array}{cc}
\lambda\left\langle g^{A} \nabla_{p e r}^{T} g^{B} \mathbf{A}\right\rangle & \lambda\left\langle\varphi^{q} \nabla_{p e r}^{T} g^{B} \mathbf{A}\right\rangle \\
\lambda\left\langle g^{A} \nabla_{p e r}^{T} \varphi^{p} \mathbf{A}\right\rangle & \left\langle\varphi^{q} \nabla_{p e r}^{T} \varphi^{p} \mathbf{A}\right\rangle
\end{array}\right]\right)\left[\begin{array}{c}
\nabla_{n p e r} \psi \\
\nabla_{n p e r} \mathbf{a}_{q}
\end{array}\right]+
\end{aligned}
$$

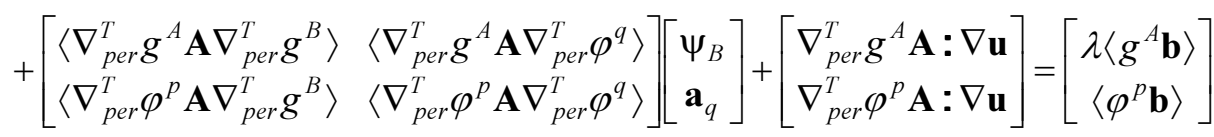

In fact, the refined tolerance model is a certain shortwave - longwave model.

In general, the composite, the elastodynamics of which is described by (12), does not have to move (in the framework of this model) impaired long- and short-wave perturbations regardless.

Unfortunately, (12) consists on infinite number of equations for an infinite number of basic unknown fields and hence in this form this model is useless.

\section{The shape functions graduation}

The drawback of the infinite number of basic unknowns in equations (12) seems to be a serious mathematical problem. Fortunately, if the finite sequence of shape functions is properly chosen, it can be graduating. This procedure boils down to introducing the infinite sequence of $n$-tuples $\left(g_{(n)}^{1}, g_{(n)}^{2}, \ldots, g^{N}(n)\right)$ enumerated by $n$ for which

$$
g^{A}(x)=\lim g_{(n)}^{A}(x)=0
$$


The graduation procedure reduces the refined model (12) to the simplest one:

$$
\begin{aligned}
& \langle\rho\rangle \ddot{\mathbf{u}}-\left\langle\nabla^{T}\{\langle\mathbf{A}\rangle[\nabla \mathbf{u}]\}-\left\langle\nabla^{T}\left\{\left\langle\mathbf{A}\left[\nabla g^{A}\right]\right\rangle \Psi_{A}\right\}\right\rangle-\left\langle\nabla^{T}\left\{\left\langle\mathbf{A}\left[\nabla \varphi^{p}\right]\right\rangle \mathbf{a}_{p}\right\}\right\rangle=\langle\mathbf{b}\rangle\right. \\
& \left(\left[\begin{array}{cc}
0 & 0 \\
0 & \left\langle\rho \bar{\varphi}^{p} \bar{\varphi}^{q}\right\rangle
\end{array}\right]\left[\begin{array}{l}
\ddot{\Psi}_{B} \\
\ddot{\mathbf{a}}_{q}
\end{array}\right]-\nabla_{\text {nper }}^{T}\left(\left[\begin{array}{cc}
0 & 0 \\
0 & \left\langle\bar{\varphi}^{p} \bar{\varphi}^{q} \mathbf{A}\right\rangle
\end{array}\right] \nabla_{n p e r}^{T}\left[\begin{array}{l}
\boldsymbol{\Psi}_{B} \\
\mathbf{a}_{q}
\end{array}\right]\right)\right)+ \\
& +\left(\left[\begin{array}{cc}
0 & 0 \\
\left\langle\bar{\varphi}^{p} \nabla_{p e r}^{T} g^{B} \mathbf{A}\right\rangle & \frac{1}{\lambda}\left\langle\bar{\varphi}^{p} \nabla_{p e r}^{T} \bar{\varphi}^{q} \mathbf{A}\right\rangle
\end{array}\right]-\left[\begin{array}{cc}
0 & \left\langle\bar{\varphi}^{q} \nabla_{p e r}^{T} g^{A} \mathbf{A}\right\rangle \\
0 & \frac{1}{\lambda}\left\langle\bar{\varphi}^{q} \nabla_{p e r}^{T} \bar{\varphi}^{p} \mathbf{A}\right\rangle
\end{array}\right]\right)\left[\begin{array}{c}
\nabla_{n p e r} \boldsymbol{\psi} \\
\nabla_{n p e r} \mathbf{a}_{q}
\end{array}\right]+ \\
& +\left[\begin{array}{cc}
\left\langle\nabla_{p e r}^{T} g^{A} \mathbf{A} \nabla_{p e r} g^{B}\right\rangle & \frac{1}{\lambda}\left\langle\nabla_{p e r}^{T} g^{A} \mathbf{A} \nabla_{p e r} \bar{\varphi}^{q}\right\rangle \\
\frac{1}{\lambda}\left\langle\nabla_{p e r}^{T} \bar{\varphi}^{p} \mathbf{A} \nabla_{p e r} g^{B}\right\rangle & \frac{1}{\lambda^{2}}\left\langle\nabla_{p e r}^{T} \bar{\varphi}^{p} \mathbf{A} \nabla_{p e r} \bar{\varphi}^{q}\right\rangle
\end{array}\right]\left[\begin{array}{l}
\psi_{B} \\
\mathbf{a}_{q}
\end{array}\right]+ \\
& +\left[\begin{array}{c}
\left\langle\nabla_{p e r}^{T} g^{A} \mathbf{A}\right\rangle \nabla \mathbf{u} \\
\frac{1}{\lambda}\left\langle\nabla_{p e r}^{T} \bar{\varphi}^{p} \mathbf{A}\right\rangle \nabla \mathbf{u}
\end{array}\right]=\left[\begin{array}{c}
0 \\
\left\langle\bar{\varphi}^{p} \mathbf{b}\right\rangle
\end{array}\right]
\end{aligned}
$$

In the next section we are to prove that two-phased laminated composite moves (in the framework of refined tolerance model) impaired long- and short-wave perturbations, regardless.

\section{Special case}

For two-phased laminated composite the Fourier basis will be introduced in the form

$$
\begin{aligned}
& \varphi^{p}\left(\frac{x}{l}\right)= \begin{cases}\frac{1}{2} \cos \frac{p \pi}{l^{I}} x, & x \in\left[0, l^{I}\right] \\
\frac{1}{2} \cos \frac{p \pi}{l^{I I}}(x-l), & x \in\left[l^{I}, l^{I I}\right]\end{cases} \\
& l=l^{I}+l^{I I}
\end{aligned}
$$

At the same time the single saw-like function will be treated as the finite sequence of shape functions $\left(g^{1}, g^{2}, \ldots, g^{N}\right)$. Here $N=1$. The rescaling of the saw-like function will be realized according to the formula, cf. Fig. 1. 


$$
\begin{aligned}
& g_{(1)}^{A}(x)=g^{A}(x) \\
& g_{(n+1)}(x)=\left\{\begin{array}{lll}
g_{(n)}(x) \text { for } & \left|g_{(n)}(x)\right| \leq \frac{1}{2} \mu_{(n)} \\
\mu_{(n)}-g_{(n)}(x) & \text { for } & \left|g_{(n)}(x)\right|>\frac{1}{2} \mu_{(n)} \\
-\mu_{(n)}-g_{(n)}(x) & \text { for } & \left|g_{(n)}(x)\right|<-\frac{1}{2} \mu_{(n)}
\end{array}\right.
\end{aligned}
$$

where

$$
\mu_{(n)} \equiv \max \left\{\left|g_{(n)}(x)\right|: x \in \Delta\right\}
$$

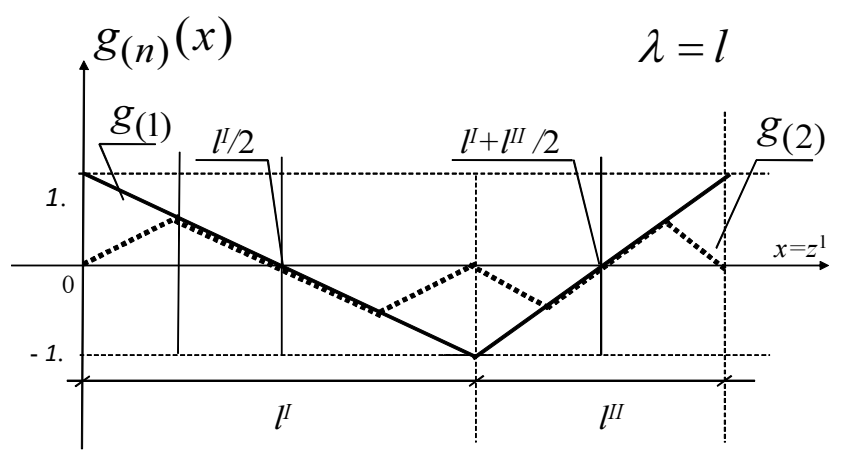

Fig. 1. The graduation of the saw-like function

In the examined case (12) $)_{3}$ takes the form

$$
\{\mathbf{A}\} \bar{\Psi}+[\mathbf{A}] \nabla_{n p e r} \mathbf{u}=0
$$

for the modified fluctuation field

$$
\bar{\psi} \equiv \psi+\left(\mathbf{a}_{1}+\mathbf{a}_{3}+\mathbf{a}_{5}+\ldots\right)
$$

This new field can be reduced from the refined model system equations and finally (12) takes the form in which the long-wave part $\mathbf{w}_{\text {long }}$ of the displacement field $\mathbf{w}$ is represented here by $\mathbf{u}$, namely $\mathbf{w}_{\text {long }}=\mathbf{u}$, and should satisfy single equation

$$
\langle\rho\rangle \ddot{\mathbf{u}}-\nabla^{T}\left(\mathbf{A}^{e f f} \nabla \mathbf{u}\right)=\langle\mathbf{b}\rangle
$$

for

$$
\begin{aligned}
& {[\mathbf{A}]_{i j k} \equiv\left[\bar{C}_{i j k}\right]=\left\langle\nabla_{n p e r}^{T} g C_{i j k 1}\right\rangle,} \\
& \{\mathbf{A}\}_{j k} \equiv\left\{\widetilde{C}_{j k}\right\}=\left\langle\nabla_{n p e r}^{T} g C_{1 j k 1} \nabla_{n p e r}^{T} g\right\rangle,
\end{aligned}
$$


while the short-wave part $\mathbf{w}_{\text {short }}$ of $\mathbf{w}$ is represented by Fourier coefficients $\mathbf{a}_{p}$, which should satisfy the separate system of equations

$$
\begin{aligned}
& \frac{\lambda^{2}}{8}\left\{\langle\rho\rangle \ddot{\mathbf{a}}_{p}-\nabla_{n p e r}^{T}\left(\langle\mathbf{A}\rangle \nabla_{n p e r} \mathbf{a}_{p}\right)\right\}_{+} \\
& +\lambda\left(\left\langle\bar{\varphi}^{p} \nabla_{n p e r}^{T} \bar{\varphi}^{q} \mathbf{A}\right\rangle-\left\langle\bar{\varphi}^{q} \nabla_{n p e r}^{T} \bar{\varphi}^{p} \mathbf{A}\right\rangle\right) \nabla_{n p e r} \mathbf{a}_{q}=\left\langle\bar{\varphi}^{p} \mathbf{b}\right\rangle
\end{aligned}
$$

where

$$
\bar{\varphi}^{p}(x) \equiv \lambda \varphi^{p}\left(\frac{x}{\lambda}\right)
$$

and the effective stiffness matrix has the well-known form

$$
\mathbf{A}^{\text {eff }}=\left[\begin{array}{ccc}
\left\langle C_{1 j k 1}\right\rangle_{H} & \left\langle C_{1 i j 2}\right\rangle & \left\langle C_{1 i j 3}\right\rangle \\
\left\langle C_{2 i j 1}\right\rangle & \left\langle C_{2 i j 2}\right\rangle & \left\langle C_{2 i j 3}\right\rangle \\
\left\langle C_{3 i j 1}\right\rangle & \left\langle C_{3 i j 2}\right\rangle & \left\langle C_{3 i j 3}\right\rangle
\end{array}\right]
$$

for $\left\langle C_{1 i j 1}\right\rangle_{H}=\left\langle C_{1 j i 1}\right\rangle-\left[\bar{C}_{i k}\right]^{T}\left\{\widetilde{C}_{1 k l 1}\right\}^{-1}\left[\bar{C}_{l j}\right], i, j, k, l=1,2,3$, but in the modeling procedure presented in this paper it has been introduced without taking into account the limit passage to the asymptotic case $\lambda \rightarrow 0$. The short-wave part of the refined model is the system of a second order differential equation with respect to the directions perpendicular to the periodicity directions and is strictly related to the analysis of the existence and finding of different solutions of the second order quadratic matrix equation, cf. [4].

\section{Final remarks}

In the paper the refined model of linear elastodynamics of periodic composites is proposed. Derivation of this model is based on the idea of the adjustment of the residual field between the displacement field and its micro-macro approximation onto a certain series with sufficiently good properties. This adjustment is realized by imposing onto a finite sequence of shape functions the additional condition of continuity of the tolerance stresses normal to the interfaces. Under this condition the residual displacement is a continuously differentiable field and hence opens the way to find solutions to the initial-boundary problems using theory of the Fourier series.

In the framework of the proposed refined model the special case of two-phased laminated composite has been examined. In this special case the effective stiffness tensor has been obtained. However, it coincides with the same tensor in the framework of other known approaches, but it is obtained here without using the limit passage to the asymptotic case. 
Moreover, two-phased laminated composite moves (in the framework of refined tolerance model) impaired long-and short-wave perturbations regardless.

The method of modeling similar to that used in the paper has been applied in [5-12].

\section{References}

[1] Woźniak C., Wierzbicki E., Averaging Techniques in Thermomechanics of Composite Solids, Częstochowa University of Technology Press, Częstochowa 2000.

[2] Woźniak C. (ed.), Developments in Mathematical Modeling and Analysis of Microstructured Media, Silesian Technical University Press, Gliwice 2010.

[3] Woźniak C. (ed.), Thermomechanics of Microheterogeneous Solids and Structures. Tolerance Averaging Approach, Lodz Technical University Press, Lodz 2009.

[4] Jędrysiak J., Termomechanika laminatów, płyt i powłok o funkcyjnej gradacji własności, Lodz Technical University Press, Lodz 2010.

[5] Kula D., Mazewska M., Wierzbicki E., Some remarks on the tolerance averaging of heat conduction in chessboard palisade-type periodic composites, Scientific Review, Engineering and Enviromental Sciences 2012, 21(3), 57, 131-140.

[6] Mazewska M., Wierzbicki E., Modelowanie tolerancyjne przewodnictwa ciepła w kompozytach o strukturze dwukierunkowo-periodycznej, Acta Scientarum Polonarum, Architectura 2013, 12(1), 3-17.

[7] Michalak B., Termomechanika ciał z pewną niejednorodną mikrostrukturą: technika tolerancyjnej aproksymacji, Lodz Technical University Press, Lodz 2010.

[8] Cielecka I., Jędrysiak J., A non-asymptotic model of dynamics of honeycomb lattice-type plates, J. Sound and Vibration 2006, 296, 130-149.

[9] Nagórko W., Wągrowska M., A contribution to modeling of composite solids, J. Theor. Appl. Mech. 2002, 40, 149-158.

[10] Wierzbicki E., Woźniak C., On the dynamic behaviour of honeycomb based composite solids, Acta Mechanica 2000, 141, 161-172.

[11] Mazewska M., Tolerance Modeling of Boundary Effect Behavior in Hexagonal-type Composites, Doctoral dissertation, Warsaw University of Life Sciences, Warsaw 2013.

[12] Woźniak M., Wierzbicki E., Woźniak C., A macroscopic model of the diffusion and heat transfer processes in a periodically micro-stratified solid layer, Acta Mechanica 2002, 157, 175-185. 